\title{
CT and MRI Findings of Glomangiopericytoma in the Head and Neck: Case Series Study and Systematic Review
}

\author{
(D) C.H. Suh, DJ.H. Lee, (D) M.K. Lee, (D)S.J. Cho, (D) S.R. Chung, (D).J. Choi, and (DJ.H. Baek
}

\begin{abstract}
SUMMARY: Glomangiopericytoma is a rare sinonasal mesenchymal tumor of borderline or low malignant potential. We reviewed the CT and MR imaging findings of head and neck glomangiopericytoma via a retrospective case series study and systematic review. Our study revealed that glomangiopericytoma is a well-defined lobulated avidly enhancing soft-tissue mass with erosive bony remodeling that is most commonly found in the sinonasal cavity. Typically, it is hyperintense on T2-weighted images with vascular signal voids, has a high mean ADC value, and a wash-in and washout pattern on dynamic contrast-enhanced MR imaging. Although the CT findings are nonspecific, typical MR imaging findings, including those on the ADC map and dynamic contrastenhanced MR imaging, may be helpful for differentiating glomangiopericytomas from other hypervascular tumors in the head and neck.
\end{abstract}

ABBREVIATION: DCE = dynamic contrast-enhanced

G lomangiopericytoma is a rare sinonasal mesenchymal tumor demonstrating a perivascular myoid phenotype and is one of the sinonasal vascular spindle cell tumors, such as spindle cell hemangioma, kaposiform hemangioendothelioma, and Kaposi sarcoma. $^{1-3}$

Glomangiopericytoma, which accounts for $<0.5 \%$ of all sinonasal tumors, was categorized as a borderline or low-malignant-potential tumor by the World Health Organization in 2005. ${ }^{1}$ A previous study showed that 18 of 104 patients $(17.3 \%)$ had a local recurrence and were managed by additional surgery. ${ }^{4}$ Glomangiopericytoma was formerly called "sinonasal hemangiopericytoma" because of a hemangiopericytomatous pattern.

To date, a number of case reports have been published describing the CT or MR imaging findings of glomangiopericytoma in the head and neck. Although these previous reports demonstrated that glomangiopericytoma is usually a well-defined and avidly enhancing soft-tissue mass with erosive remodeling mainly in the sinonasal cavity, there has been no report systematically

Received April 26, 2019; accepted after revision October 7

From the Department of Radiology and Research Institute of Radiology, University of Ulsan College of Medicine, Asan Medical Center, Seoul, Republic of Korea.

Please address correspondence to Jeong Hyun Lee, MD, PhD, Department of Radiology and Research Institute of Radiology, University of Ulsan College of Medicine, Asan Medical Center, 86 Asanbyeongwon-Gil, Songpa-Gu, Seoul 05505, Republic of Korea; e-mail: jeonghlee@amc.seoul.kr

Indicates article with supplemental on-line tables.

http://dx.doi.org/10.3174/ajnr.A6336 reviewing the $\mathrm{CT}$ and MR imaging findings of glomangiopericytoma in the head and neck. In addition, details on the findings on advanced MR imaging methods including DWI and dynamic contrast-enhanced (DCE)-MR imaging have not yet been published.

Therefore, the purpose of our study was to review the CT and MR imaging findings of glomangiopericytoma in the head and neck, including findings on DWI and DCE-MR imaging, via a retrospective study of a case series and a systematic review.

\section{Case Series}

Our study was approved by the institutional review board of Asan Medical Center, and the requirement for informed consent was waived due to the retrospective nature of the study. The pathology reports for January 2010 to January 2019 were electronically searched using "glomangiopericytoma," "sinonasal hemangiopericytoma," "sinonasal-type hemangiopericytoma," "hemangiopericytoma-like tumor," and "hemangiopericytoma of sinonasal origin" as keywords. Patients who underwent preoperative CT and/or MR imaging were enrolled. Clinical and demographic data including age, sex, and symptoms were acquired from the electronic medical record. The imaging findings including location, size, border, adjacent bone change, CT density, enhancement pattern, MR imaging signal intensity on T2WI, $\mathrm{ADC}$ value of the whole tumor, the presence of vascular signal voids, and DCE-MR imaging patterns were independently reviewed by 2 board-certificated neuroradiologists (J.H.L., with 

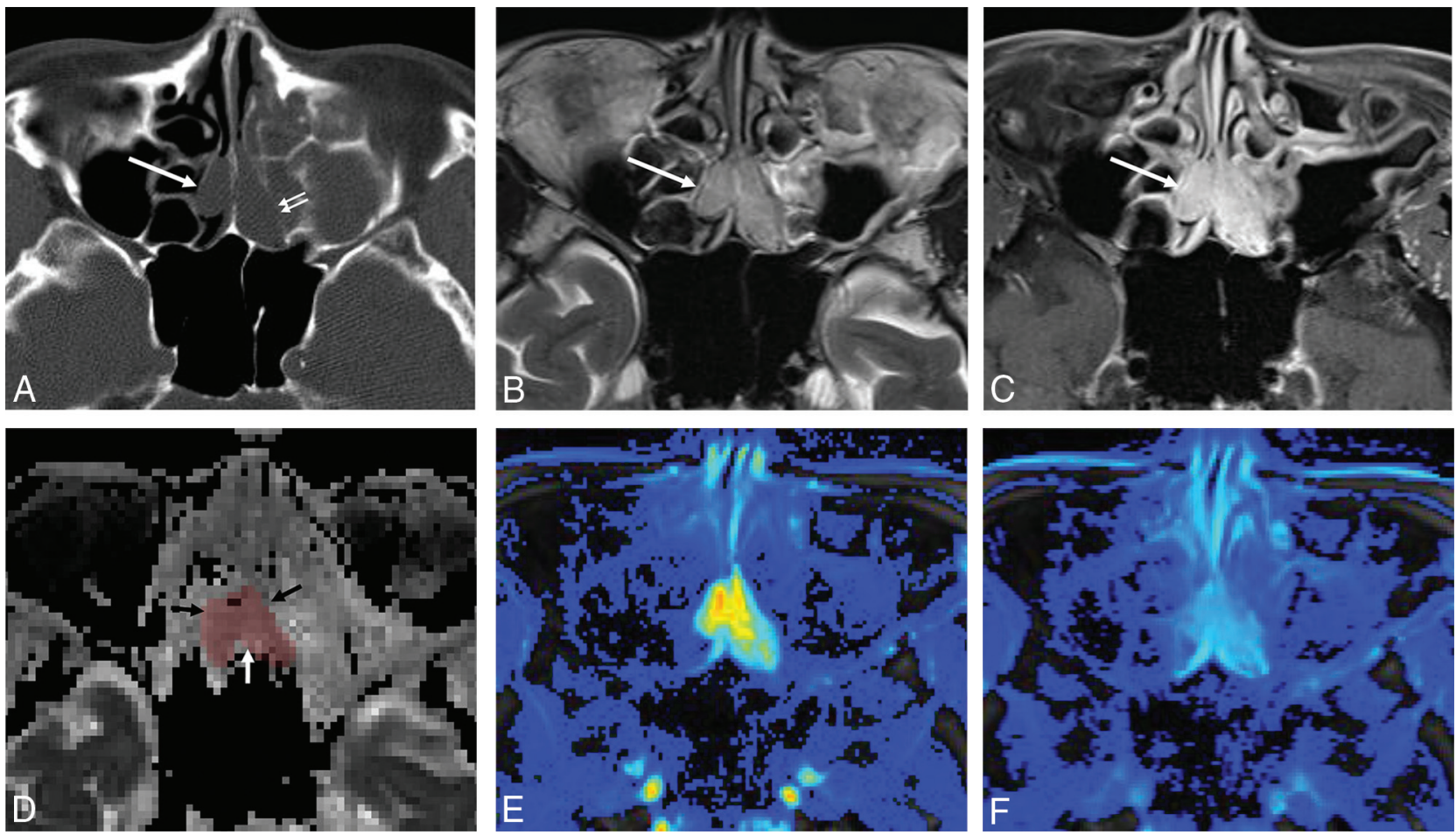

FIG 1. A 79-year-old woman with sinonasal glomangiopericytoma. A, Nonenhanced CT shows a midline bilobed soft-tissue mass (long arrow) overriding the permeated nasal septum with erosive remodeling on the bony wall of the left ethmoidal sinus (short arrows). On MR imaging, the mass (arrow) shows slight hyperintensity on T2WI $(B)$ and strong and homogeneous enhancement on fat-suppressed contrast-enhanced TIWI (C). D, On the ADC map, the mass shows a high mean ADC value $\left(1.47 \times 10^{-3} \mathrm{~mm}^{2} / \mathrm{s}\right)$ (arrows). E and $F$, On DCE-MR imaging, the mass reveals wash-in and washout patterns on the initial and final area under the curve maps, which were obtained by trapezoidal integration of the normalized signal intensity curve during the initial 90 seconds after the onset of contrast enhancement and during the last 90 seconds using the same enhancing voxels.

18 years of experience in head and neck neuroradiology, and C.H.S., with 6 years of experience in neuroradiology). We placed ROIs for the entire tumor to obtain a mean ADC value of the whole tumor.

Six patients satisfied the inclusion criteria ( 3 men and 3 women; mean age, 67 years; range, $48-79$ years; On-line Table 1 ). Four patients had symptoms, and 2 patients were identified incidentally on imaging studies. Following initial detection, all patients underwent an operation. One patient had preoperative embolization of the tumor. Two patients had minor bleeding during resection, but the other 3 had no bleeding complication, despite no preoperative embolization. Four glomangiopericytomas were located in the sinonasal cavity; 1 , in the masticator/buccal spaces, and 1 , in the prevertebral space. The maximum diameters ranged from 2.9 to $5 \mathrm{~cm}$ (median, $3.3 \mathrm{~cm}$ ).

All patients underwent CT, which was performed using $\geq 16$ detector row CT scanners with excellent image quality. Intravenous iodine contrast agent was used in all except 1 patient. All glomangiopericytomas were well-defined round or lobulated contoured soft-tissue masses (Fig 1). Five tumors were in direct contact with the adjacent bone, which showed erosive remodeling, while 1 tumor was surrounded by soft tissue. On contrastenhanced CT, 2 tumors revealed avid and homogeneous enhancement patterns that were slightly lower than the adjacent vessels (Fig 2), whereas 3 tumors demonstrated heterogeneous enhancement.
Four of 6 patients underwent MR imaging, which included DWI in 1 patient and both DWI and DCE-MR imaging in 2 patients. All MR imaging was performed using a 3T scanner with excellent image quality. Detailed information about our own MR imaging parameters was the same as that published in previous articles by our group. ${ }^{5,6}$

On MR imaging, 2 glomangiopericytomas showed homogeneous hyperintensity on T2WI, 1 glomangiopericytoma showed intermediate signal intensity, and 1 glomangiopericytoma showed heterogeneous intermediate-to-high signal intensity. All glomangiopericytomas revealed vascular signal voids on T2WI. The 3 patients for whom ADC maps were available demonstrated high mean ADC values ranging from 1.27 to $2.09 \times 10^{-3} \mathrm{~mm}^{2} / \mathrm{s}$ (On-line Table 1 and Figs 1 and 2). On contrast-enhanced MR imaging, 3 glomangiopericytomas in the sinonasal cavity or masticator space revealed avid and homogeneous enhancement, whereas 1 glomangiopericytoma in the prevertebral space revealed heterogeneous enhancement. On DCE-MR imaging, 2 glomangiopericytomas revealed a rapid wash-in and washout pattern (Fig 1).

For the systematic review, a systematic search of PubMed and EMBASE was performed using the following search term: ((glomangiopericytoma) OR ("sinonasal hemangiopericytoma") OR ("sinonasal-type hemangiopericytoma") OR ("hemangiopericytoma-like tumor") OR ("hemangiopericytoma of sinonasal origin")) AND ((“computed tomography") OR (CT) OR ("magnetic 

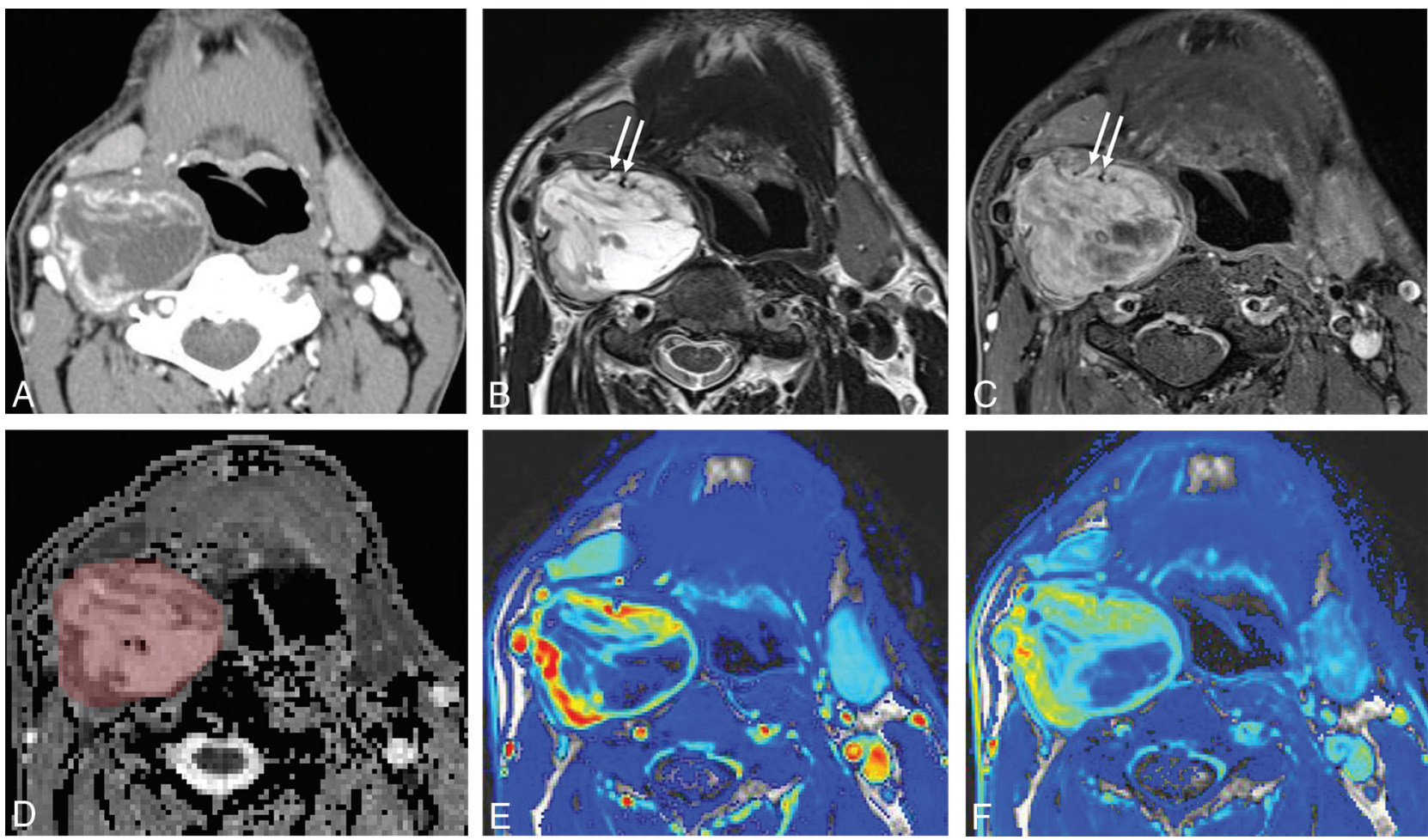

FIG 2. A 72-year-old man with glomangiopericytoma in the prevertebral space. $A$, Contrast-enhanced CT shows a well-defined heterogeneously enhancing mass in the right prevertebral space. The mass demonstrates iso- to hyperintensity on T2WI (B) and has heterogeneous enhancement on fat-suppressed contrast-enhanced TIWI (C). Note several vascular signal voids (arrows) at the periphery of the mass. $D$, On the ADC map, the mass shows a high mean ADC value $\left(2.09 \times 10^{-3} \mathrm{~mm}^{2} / \mathrm{s}\right)$. E and $F$, On DCE-MR imaging, the mass reveals rapid washin and washout patterns on the initial and final area under the curve maps, which were obtained by trapezoidal integration of the normalized signal intensity curve during the initial 90 seconds after the onset of contrast enhancement and during the last 90 seconds using the same enhancing voxels.

resonance imaging") OR (“MR imaging") OR (MRI)). The literature search was not limited by any filters or published language. The search was updated until August 15, 2019. The literature search, study selection, and data extraction were independently performed by 2 board-certificated neuroradiologists (J.H.L. and C.H.S.)

This systematic search originally yielded 59 articles (38 from PubMed and 21 from EMBASE). After removal of duplicate articles and those that were not in the field of interest, 11 case reports with 14 cases were left for inclusion in this systematic review (On-line Table 2). ${ }^{7-17}$ Eleven of 14 glomangiopericytomas were located in the sinonasal cavity and had maximum diameters ranging from 2.9 to $4 \mathrm{~cm}$. On CT, 11 glomangiopericytomas were well-defined lobulated contoured soft-tissue masses and 7 showed erosive remodeling of adjacent bone. On contrast-enhanced CT, 6 glomangiopericytomas revealed strong and homogeneous enhancement patterns, while 2 glomangiopericytomas demonstrated heterogeneous enhancement. On MR imaging, 3 glomangiopericytomas showed hyperintensity on T2WI. These CT and MR imaging findings were similar to our findings.

\section{DISCUSSION}

Both our case series and the systematic review confirmed that glomangiopericytomas are well-defined round or lobulated, contoured soft-tissue masses that frequently show erosive bone remodeling, especially in the sinonasal cavity. Our study also revealed that glomangiopericytomas usually show avid and homogeneous enhancement on contrast-enhanced CT or MR imaging, intermediate-to-high signal intensity on T2WI, high ADC values, and rapid wash-in and washout patterns on DCE-MR imaging. They are also accompanied by vascular signal voids on T2WI. Although the individual imaging findings might be nonspecific, a combination of these findings on CT and MR imaging, especially when DWI and DCE-MR imaging are included, may help to suggest glomangiopericytoma of borderline malignant potential, which is important in preoperative planning of the surgical approach or extent of resection.

In this study, we report on the $\mathrm{CT}$ and MR imaging (including DWI and DCE-MR imaging) findings of glomangiopericytoma. While the lesions are imaged in a manner similar to that of other vascular tumors, the combination of findings may help differentiate glomangiopericytoma from other hypervascular tumors. High $A D C$ values indicate low cellularity, which reflects the low malignant potential of the tumor. Therefore, high ADC values may be a key imaging finding for differentiating glomangiopericytoma from malignant sinonasal cancer. ${ }^{18}$ The signal voids on T2WI and avid enhancement suggest a vascular tumor. Surgeons may request preoperative embolization in this category of lesion before endoscopic resection. ${ }^{9}$ 
Given the submucosal nature of glomangiopericytomas, they can be differentiated from more common mucosal tumors such as squamous cell carcinomas or inverted papillomas. The differential diagnosis for a submucosal tumor is broad and can include solitary fibrous tumor, lobular capillary hemangioma, or nasopharyngeal angiofibroma. Differentiating these entities when they occur in the head and neck can be challenging because of similar imaging findings. However, the distinction is clinically important because glomangiopericytoma has borderline or low malignant potential, unlike some of the other disease entities. Solitary fibrous tumor, formerly known as hemangiopericytoma, consists of a cellular spindle cell proliferation effacing submucosal structures with staghorn-type vessels, ${ }^{19}$ with the tumors being characterized by chromosomal translocation resulting in the formation of a NAB2-STAT6 fusion gene; thus nuclear staining of STAT6 is a reference standard method for diagnosing solitary fibrous tumors. ${ }^{20}$ Glomangiopericytoma is most commonly confused with solitary fibrous tumor because solitary fibrous tumor is also a well-defined tumor with avid enhancement and a washout time-intensity curve pattern on DCE-MR imaging. ${ }^{21}$ However, solitary fibrous tumor in the sinonasal cavity shows iso- to hypointensity on T2WI because of its fibrous component. ${ }^{22}$ Although it is difficult to differentiate glomangiopericytoma from solitary fibrous tumor on CT, these signal intensity differences on T2WI may be helpful.

Lobular capillary hemangioma is another potential differential diagnosis; it has a lobular architecture with staghorn-type vessels in the interlobular stroma. ${ }^{23}$ Although lobular capillary hemangioma is also known to be a well-defined avidly-enhancing mass with hyperintensity on T2WI and a washout time-intensity curve pattern, bony changes are not usually seen on CT or MR imaging. ${ }^{24,25}$ Nasopharyngeal angiofibroma has abundant stromal collagen and a prominent vascular stroma. Although angiofibroma is a similar hypervascular tumor, the typical demographic data, including age and sex and the characteristic location (ie, lateral nasopharyngeal mucosa at the sphenopalatine foramen), can help to differentiate angiofibroma from glomangiopericytoma. When glomangiopericytoma occurs outside the sinonasal cavity, neurogenic tumors can be one of the major differential diagnoses. However, neurogenic tumor can be differentiated by its location, typical shape, and heterogeneous hypoenhancement.

The main limitations of our study are the small number of enrolled cases and its retrospective nature, inevitable given the rare occurrence of glomangiopericytoma in the head and neck. We have attempted to overcome these limitations by performing a systematic review, and the results are in accord with ours. We were able to include 3 cases with advanced MR imaging techniques, and these techniques may be of assistance in generating a more focused differential diagnosis. Tissue sampling remains the standard, however. We believe that our results could provide an insight into further investigation of glomangiopericytoma with advanced MR imaging techniques.

\section{CONCLUSIONS}

Our case series and the systematic review revealed that glomangiopericytoma is a well-defined lobulated avidly-enhancing softtissue mass with erosive bony remodeling usually occurring in the sinonasal cavity. It typically shows hyperintense signal on T2WI with vascular signal voids, a high mean ADC value, and a wash-in and washout pattern on DCE-MR imaging. Although the CT findings are nonspecific, typical MR imaging findings, including the ADC value and DCE-MR imaging pattern, can be helpful for differentiating glomangiopericytomas from other hypervascular tumors in the head and neck, especially in the sinonasal cavity.

Disclosures: Jung Hwan Baek—UNRELATED: Consultancy: radiofrequency ablation, Comments: consultant of 2 radiofrequency companies, StarMed and RF Medical since 2017.

\section{REFERENCES}

1. Stelow EB, Bishop JA. Update from the 4th Edition of the World Health Organization Classification of Head and Neck Tumours: Tumors of the Nasal Cavity, Paranasal Sinuses and Skull Base. Head Neck Pathol 2017;11:3-15 CrossRef Medline

2. Compagno J, Hyams VJ. Hemangiopericytoma-like intranasal tumors: a clinicopathologic study of 23 cases. Am J Clin Pathol 1976;66:672-83 CrossRef Medline

3. Marusic Z, Billings SD. Histopathology of spindle cell vascular tumors. Surg Pathol Clin 2017;10:345-66 CrossRef Medline

4. Thompson LD, Miettinen M, Wenig BM. Sinonasal-type hemangiopericytoma: a clinicopathologic and immunophenotypic analysis of 104 cases showing perivascular myoid differentiation. Am J Surg Pathol 2003;27:737-49 CrossRef Medline

5. Choi YJ, Lee JH, Kim HO, et al. Histogram analysis of apparent diffusion coefficients for occult tonsil cancer in patients with cervical nodal metastasis from an unknown primary site at presentation. Radiology 2016;278:146-55 CrossRef Medline

6. Lee JY, Cheng KL, Lee JH, et al. Detection of local recurrence in patients with head and neck squamous cell carcinoma using voxelbased color maps of initial and final area under the curve values derived from DCE-MRI. AJNR Am J Neuroradiol 2019;40:13921401 CrossRef Medline

7. Kono M, Bandoh N, Matsuoka R, et al. Glomangiopericytoma of the nasal cavity with CTNNB1 p.S37C mutation: a case report and literature review. Head Neck Pathol 2019;13:298-303 CrossRef Medline

8. Anschuetz L, Buchwalder M, Dettmer M, et al. A clinical and radiological approach to the management of benign mesenchymal sinonasal tumors. ORL J Otorhinolaryngol Relat Spec 2017;79:131-46 CrossRef Medline

9. Psoma E, Karkos PD, Dova S, et al. Sinonasal glomangiopericytoma treated with preoperative embolisation and endoscopic sinus surgery. Ecancermedicalscience 2016;10:692 CrossRef Medline

10. Kim J, Jeon J, Kim DH, et al. Glomangiopericytoma and glomus tumor of the sinonasal tract: a report of two cases with emphasis on the differential diagnosis. Pathol Int 2016;66:348-50 CrossRef Medline

11. Fox DP, Helekar BS, Gallagher KK. Laryngeal, retropharyngeal, and cervical glomangiopericytomas: a case report. Otolaryngol Head Neck Surg 2016;155:1059-60 CrossRef Medline

12. Papierska L, Cwikła JB, Misiorowski W, et al. FGF23 producing mesenchymal tumor. Case Rep Endocrinol 2014;2014:492789 CrossRef Medline

13. Arpaci RB, Kara T, Vayisoğlu Y, et al. Sinonasal glomangiopericytoma. J Craniofac Surg 2012;23:1194-96 CrossRef Medline

14. Terada T, Kato T. Sinonasal-type hemangiopericytoma of the nasal cavity and paranasal sinus. Int J Clin Oncol 2012;17:169-73 CrossRef Medline

15. Conrad GR, Sinha P, Absher KJ. FDG PET/CT findings of a glomangiopericytoma. Clin Nucl Med 2011;36:462-64 CrossRef Medline 
16. Taglialatela Scafati C, D'Antonio A, Taglialatela Scafati S, et al. Glomangiopericytoma of the pterygomandibular space: an unusual case. Br J Oral Maxillofac Surg 2007;45:673-75 CrossRef Medline

17. Palacios E, Restrepo S, Mastrogiovanni L, et al. Sinonasal hemangiopericytomas: clinicopathologic and imaging findings. Ear Nose Throat J 2005;84:99-102 CrossRef Medline

18. Gencturk M, Ozturk K, Caicedo-Granados E, et al. Application of diffusion-weighted MR imaging with ADC measurement for distinguishing between the histopathological types of sinonasal neoplasms. Clin Imaging 2019;55:76-82 CrossRef Medline

19. Thompson LD. Sinonasal tract glomangiopericytoma (hemangiopericytoma). Ear Nose Throat J 2004;83:807 Medline

20. Anzai T, Saito T, Tsuyama S, et al. A case of glomangiopericytoma at the nasal septum. Head Neck Pathol 2018;12:572-75 CrossRef Medline
21. Ganly I, Patel SG, Stambuk HE, et al. Solitary fibrous tumors of the head and neck: a clinicopathologic and radiologic review. Arch Otolaryngol Head Neck Surg 2006;132:517-25 CrossRef Medline

22. Yang BT, Song ZL, Wang YZ, et al. Solitary fibrous tumor of the sinonasal cavity: CT and MR imaging findings. AJNR Am J Neuroradiol 2013;34:1248-51 CrossRef Medline

23. Higashi K, Nakaya K, Watanabe M, et al. Glomangiopericytoma of the nasal cavity. Auris Nasus Larynx 2011;38:415-17 CrossRef Medline

24. Yang BT, Li SP, Wang YZ, et al. Routine and dynamic MR imaging study of lobular capillary hemangioma of the nasal cavity with comparison to inverting papilloma. AJNR Am J Neuroradiol 2013;34:2202-07 CrossRef Medline

25. Kim JH, Park SW, Kim SC, et al. Computed tomography and magnetic resonance imaging findings of nasal cavity hemangiomas according to histological type. Korean J Radiol 2015;16:566-74 CrossRef Medline 